\title{
An Organizing Center for Thermohaline Excitability
}

\author{
Jan Abshagen And Axel Timmermann \\ Leibniz-Institute of Marine Science (IfM-Geomar), University of Kiel, Kiel, Germany
}

(Manuscript received 3 April 2003, in final form 19 May 2004)

\begin{abstract}
The bifurcation behavior of a conceptual heat-salt oscillator model is analyzed by means of numerical continuation methods. A global (homoclinic) bifurcation acts as an organizing center for the dynamics of the simplified convective model. It originates from a codimension-2 bifurcation in an extended parameter space. Comparison with earlier work by Cessi shows that the intriguing stochastic thermohaline excitability can be understood from the bifurcation structure of the model. It is argued that global bifurcations may play a crucial role in determining long-term variability of the thermohaline circulation.
\end{abstract}

\section{Introduction}

Analysis of recent paleorecords (Dansgaard et al. 1984; Heinrich et al. 1988; Johnsen et al. 1992; Bond et al. 1993; Bond and Lotti 1995; Severinghaus and Brook 1999) from the "Last Glacial Period" has revealed the existence of abrupt millennial-scale climate swings, known as Dansgaard-Oeschger cycles. Several climate modelers have attempted to simulate and understand these notable climate transitions (Sakai and Peltier 1997; Ganopolski and Rahmstorf 2001, 2002; Timmermann et al. 2003). A common result is that millennial-scale variability originates from a convectionadvection-diffusion oscillation, which bears many similarities with the deep-decoupling mode described by Winton (1993). Such oscillations can be excited by moderate steady or transient freshwater forcing (Sakai and Peltier 1997; Ganopolski and Rahmstorf 2001; Timmermann et al. 2003) and/or by stochastic freshwater fluctuations (Ganopolski and Rahmstorf 2002; Timmermann et al. 2003). However, the origin of the millennial-scale period has not been understood sufficiently. In Timmermann et al. (2003) it is argued that a homoclinic bifurcation and a specific type of noise excitation (coherence resonance) might have an important influence on the periodicity of the simulated millennialscale climate swings. This issue will be studied here using a conceptual two-box model (Welander 1982; Cessi 1996), capturing convective adjustment and a simple relaxation toward a reference density profile. Conceptual box models of thermohaline flow have been used

Corresponding author address: Dr. Jan Abshagen, Ocean Circulation and Climate/Theory and Modelling, Leibniz-Institute of Marine Science, University of Kiel, Düsternbrooker Weg 20, 24105 Kiel, Germany.

E-mail: jabshagen@ifm-geomar.de widely (e.g., Stommel et al. 1961; Winton 1993; Cessi 1994; Marotzke and Stone 1995; Paillard 1995; Cessi 1996, Lohmann et al. 1996; Kuhlbrodt et al. 2001; Rahmstorf 2002; Titz et al. 2001, 2002; Timmermann and Lohmann 2001; Gildor and Tziperman 2001; Schulz et al. 2002) in order to study first-order processes and feedbacks that govern the stability and variability of thermohaline flows. These simplified models, which treat the thermohaline circulation (THC) in terms of a few advectively/diffusively and/or convectively connected homogenous reservoirs, facilitate a systematic study of nonlinearities, multiple equilibria, and bifurcations. In an attempt to connect results from simplified box models and more complex zonally averaged models for the THC, Dijkstra (2000) showed that the existence of lateral advective multiple flow equilibria can be found in all levels of model complexity. Hence, simple box models are useful in order to understand dynamical features simulated by more complex models. The role of vertical processes on the other hand, such as convection, vertical diffusion, and diapycnal mixing, in generating nonlinear THC dynamics is less clear. An example of highly nontrivial dynamics resulting from a simple convective two-box model has been described by Welander (1982). Welander argues that freshwater fluxes, which force vertical convective processes, may result in self-sustained oscillations in a simple heat-salt oscillator model. An analysis of the stochastically excited convective two-box model (Welander 1982) by Cessi (1996) revealed furthermore that noise plays an important role in determining the return time of convective flushes. This behavior is consistent with the concept of coherence resonance, proposed by Pikovsky and Kurths (1997) and Lindner and Schimansky-Geier (1999). Coherence resonance has also been identified in more complex climate models (Ganopolski and Rahms- 
torf 2002; Timmermann et al. 2003) and might play a role in the generation of Dansgaard-Oeschger events.

The goal of this paper is to explain the major findings of Cessi (1996) using dynamical systems analysis.

- Freshwater fluxes determine whether the system oscillates or settles into a steady state. The period of the oscillations is very sensitive to the freshwater flux and may become arbitrarily long near the transition from steady to periodic behavior.

- The oscillations are of finite amplitude, even just past the threshold value that separates periodic behavior from a steady equilibrium.

- One extremum of the oscillation is close to the value of the steady state that exists in the absence of oscillating behavior.

- When the deterministic system reaches a steady state, oscillations can be excited easily by adding a stochastic component to the freshwater flux. The period of the resulting oscillations decreases with increasing noise amplitude, while the amplitude of the oscillation is insensitive to the amplitude of the noise.

We perform a numerical bifurcation analysis (Doedel and Kernévez 1986) of the nonlinear heat-salt oscillator (Welander 1982; Cessi 1996). We show that the four aforementioned properties of the heat-salt oscillations can be explained based on the underlying bifurcation structure of the model, namely, the existence of a homoclinic bifurcation. Moreover, we find that the homoclinic bifurcation originates from a multiple (codimension 2) bifurcation point that is, an "organizing center."

\section{Welander's heat-salt oscillator}

The model under consideration was orignally studied by Welander (1982), and a detailed description can be found in Cessi (1996). The model consists of two boxes on top of each other, each having a well-mixed temperature and salinity. The bottom box is assumed to be much deeper than the top box, and so its salinity $S_{0}$ and temperature $T_{0}$ are assumed to be constant. Temperature $T$ and the salinity $S$ of the top box change according to

$$
\begin{aligned}
& \dot{T}=-\gamma\left(T-T_{A}\right)-\kappa\left(T-T_{0}\right) \text { and } \\
& \dot{S}=\frac{F}{H} S_{0}-\kappa\left(S-S_{0}\right) .
\end{aligned}
$$

Buoyance flux is parameterized in terms of mixed boundary conditions: a relaxation condition to a prescribed atmospheric equilibrium temperature $T_{A}$ with a time constant $\gamma^{-1}$ and a prescribed salinity flux $(F / H) S_{0}$; $H$ denotes the depth of the upper box. The density $\rho(T$, $S$ ) is diagnosed from a linear equation of state:

$$
\rho(T, S)=\rho_{0}\left[1+\alpha_{S}\left(S-S_{0}\right)-\alpha_{T}\left(T-T_{0}\right)\right] .
$$

Throughout this paper we will use the nondimensionalized version of Eqs. (1) and (2) introduced by Cessi
(1996) (see also this reference for the corresponding parameter values):

$$
\dot{x}=1-x-\nu x \text { and } \dot{y}=\mu-\nu y,
$$

with the definitions

$$
x \equiv \frac{T-T_{0}}{T_{A}-T_{0}} \quad \text { and } \quad y \equiv \frac{\alpha_{S}\left(S-S_{0}\right)}{\alpha_{T}\left(T_{A}-T_{0}\right)}
$$

and the normalized "diffusion" coefficient $\nu=\kappa / \gamma$. The ratio of surface salinity and temperature flux is given by

$$
\mu=\frac{F \alpha_{s} S_{0}}{\gamma H \alpha_{T}\left(T_{A}-T_{0}\right)} .
$$

For a fixed atmospheric temperature $T_{A}$ changes in $\mu$ represent changes in the normalized freshwater flux. For constant diffusivity $\nu$ the system relaxes quickly to a stationary solution. Interesting solutions occur when we assume a stratification dependence of the diffusion coefficient $\nu=\nu[\rho(T, S)]$ (Welander 1982). For a stable stratification we assume a small value of $\nu_{1}=0.1$, representing a "nonconvective state," whereas $\nu$ attains a value of $\nu_{2}=5.0$ when the stratification becomes unstable.

The model we use throughout this work differs from the original Welander model in that it uses a generalized dependence of $\nu$ on the vertical density gradient. Instead of a Heavyside function used by Welander (1982) and Cessi (1986), a smooth approximation is used:

$$
\nu=\nu_{1}+\left(\nu_{2}-\nu_{1}\right) \frac{1+\tanh [\sigma(y-x-\varepsilon)]}{2} .
$$

The parameter $\varepsilon$ has already been introduced by Welander (1982). The additional parameter $\sigma$ represents the smoothness between the nonconvective and the "convective" state transitions. In a sense, our model can be seen as a generalization of the original Welander model with an additional parameter $\sigma$ giving rise to a more complex stratification dependence of the diffusivity $\nu(\sigma, \varepsilon)$. Note that the original discontinous model is contained in our analysis as a limiting case, $\sigma \rightarrow \infty$.

The freshwater forcing $\mu$, as in many other studies, is chosen as a natural bifurcation parameter of the system.

\section{Results}

A typical bifurcation diagram of Welander's heat-salt oscillator model is shown is Fig. 1. It captures the dependence of the normalized temperature variable $x$ on the normalized freshwater flux $\mu$. The diagram is obtained by numerical continuation (Doedel and Kernévez 1986) of the steady and time-periodic solutions following the $\mu$ variable. The parameters $\sigma$ and $\varepsilon$ are fixed to $\sigma=8$ and $\varepsilon=0.21$.

For a normalized freshwater flux $\mu$ below the Hopf bifurcation point the solid line represents the only so- 


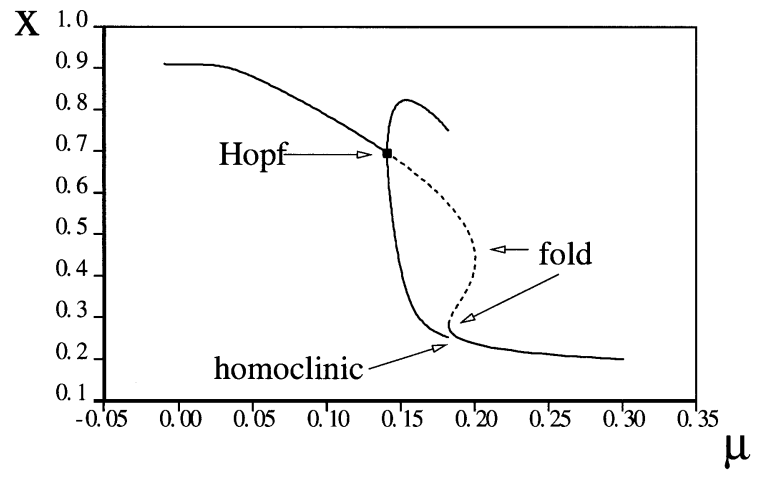

FIG. 1. Typical bifucation diagram of the Welander oscillator with freshwater forcing $\mu$. Mixing parameters $\varepsilon$ and $\sigma$ are held fixed. Selfsustained oscillation and multiple equilibria occur at "Hopf" and "fold" bifurcation points, respectively. Extrema of oscillation amplitude in $x$ are plotted with $\mu$. Periodic orbit merges with saddle point at homoclinic connection. Note that this is not directly visible because of projection on $x$ variable.

lution of the conceptual heat-salt oscillator. It is a stable fixed point, which becomes unstable via a supercritical Hopf bifurcation. The $\mu$ dependence of the emerging limit cycle is indicated in Fig. 1 by depicting also the oscillation maxima and minima. The location of the unstable fixed point arising at the Hopf bifurcation point is represented by the dashed line in Fig. 1. At a fold bifurcation point this unstable fixed point merges with a saddle point. For lower $\mu$ values of about 0.18 this saddle point merges with a third equilibrium solution in a second fold bifurcation. This third fixed point is stable for $\mu>0.2$, as indicated by the solid line, and represents a nonconvective situation forced by strong freshwater forcing. Within the parameter region in between the two fold bifurcations the Welander model exhibits three equilibria, giving rise to hysteresis behavior. However, only one of these equilibria is stable. If $\mu$ varies between, say, 0.14 and 0.25 , another type of hysteresis can arise that connects a stationary stable state with a stable oscillatory solution. For $\mu \approx 0.18$ we observe that the stable limit cycle merges with the saddle point giving rise to a homoclinic bifurcation. ${ }^{1}$ The disappearence of a limit cycle with finite amplitude, as indicated in Fig. 1, is typical for homoclinic bifurcations and represents a simple interpretation of the results discussed in Cessi (1996). Figure 2 shows the typical divergent behavior of the oscillation period near a homoclinic bifurcation. At the Hopf bifurcation point the period $T$ is entirely determined by the imaginary part of the critical eigenvalues. It is shown in Fig. 2 that this period is almost unchanged for a wide range of forcing amplitudes $\mu$. Close to the homoclinic bifurcation the period $T$ diverges quickly. This is due to the fact that close to a homoclinic bifurcation the oscillation period is no longer determined by the eigen-

\footnotetext{
${ }^{1}$ Note that because of the projection on the $x$ variable the homoclinic bifurcation point is not directly visible in Fig. 1.
}

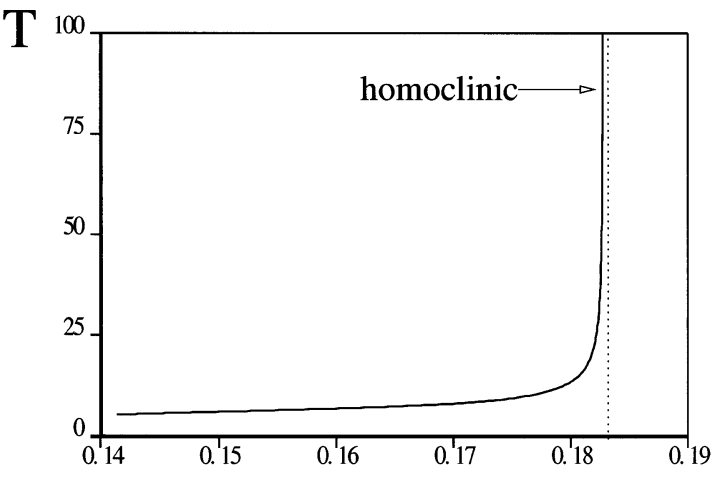

$\mu$

FIG. 2. Period $T$ of self-sustained oscillation corresponding to bifurcation diagram shown in Fig. 1. Divergence of period occurs close to homoclinic connection.

values but rather by the distance in phase space between limit cycle and saddle point. This behavior of the system operating in the parameter vicinity of a homoclinic orbit may be important in order to generate long-term climate variability, which is not understandable simply in terms of linear dynamics and linear eigenmodes, a point that has also been stressed by Timmermann et al. (2003).

A homoclinic orbit originates from a connection between a saddle point and a limit cycle. The saddle point originates from a fold bifurcation, whereas the limit cycle is born from a Hopf bifurcation. In many cases the underlying codimension- 1 bifurcations themselves result from codimension-2 bifurcations (Guckenheimer and Holmes 1983). The question we address here is whether the existing fold and Hopf bifurcations can be traced back to an organizing codimension- 2 bifurcation. In order to study this we have computed a two-parameter stability diagram of the location of fold and Hopf bifurcation points (Fig. 3). Here, $\mu$ and $\varepsilon$ are both taken as control parameters while $\sigma$ is held fixed.

The two fold lines depicted in Fig. 3 correspond to

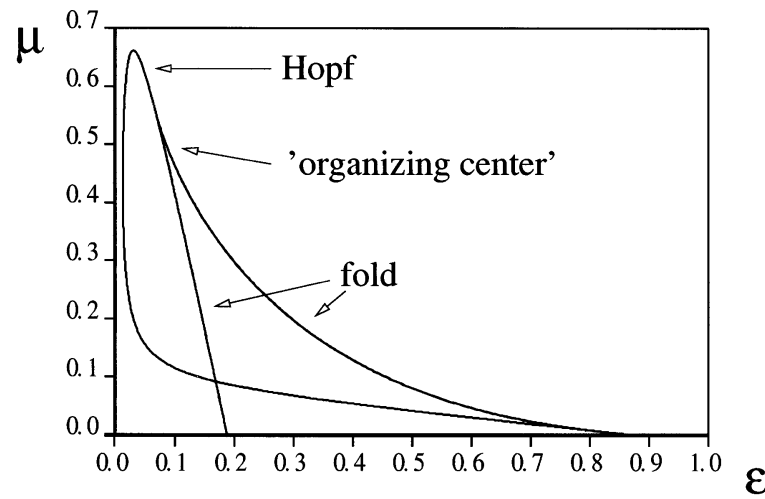

FIG. 3. Typical stability diagram of the Welander oscillator with freshwater forcing $\mu$ and mixing parameter $\varepsilon ; \sigma$ is held fixed. Fold bifurcations merge with an inverse Hopf bifurcation in multiple bifurcation points known as "organizing centers," resulting in global bifurcations. 
fold bifurcations as shown, for example, in Fig. 1 for fixed $\varepsilon$. They form a region of coexistence where multiple equilibria exist. Reducing $\varepsilon$ it is found that the two fold lines merge into a cusp bifurcation point. A further decrease of $\varepsilon$ from the cusp gives rise to an inverse Hopf bifurcation. The oscillation frequency of the limit cycle, which results from the Hopf bifurcation, is zero at the cusp point but increases with decreasing $\varepsilon$. The Hopf bifurcation is supercritical, so the amplitude is zero at onset but increases continously while reducing $\mu$. At the point where the both fold lines meet the Hopf line two real critical eigenvalues occur giving rise to a multiple bifurcation point. Such a point (in this case a Bogdanov-Takens point) is called an "organzing center." The importance of such an organizing center results from the fact that the entire bifurcation structure close to this point, and thus the observed dynamics, is completely determined by the properties of this point. Furthermore, it is known that a homoclinic bifurcation, as found in the bifurcation diagram shown in Fig. 1, emerges from such codimension-2 points (Guckenheimer and Holmes 1983). The location of the homoclinic bifurcation shown in Fig. 1 is not calculated explicitly in the two-dimensional parameter plane, but for mathematical reasons it is known that the homoclinic bifurcation originates also from the codimension-2 point. Moreover, the frequency of the homoclinic orbit is always zero, but the amplitude reduces from a finite value at larger $\varepsilon$ to zero while approaching the codimension- 2 point by decreasing $\varepsilon$. Thus it can be concluded that the qualitative bifurcation structure, and thus the dynamics of the generalized Welander oscillator, can be understood entirely from the behavior close to a single multiple bifurcation point, that is, an "organizing center." Note that a second multiple bifurcation point occurs but only for large $\varepsilon$; therefore it is not considered here.

In order to analyze the stability behavior with respect to $\sigma$ several stability diagrams similar to that shown in Fig. 3 have been computed. It is found that qualitatively the stability behavior remains the same for $\sigma \geq 8$. However, this value is associated with a rather smooth switch from convective to nonconvective behavior. In order to make a connection with the limiting case of the original discontinous Welander oscillator the stability behavior is analyzed for $\sigma$ values of up to $\sigma \leq 500$. The numerical analysis suggests that the location of the organizing center tends to $\varepsilon \rightarrow 0$ as $\sigma \rightarrow \infty$. Thus it seems reasonable to regard the bifurcation diagram shown in Fig. 1 as a smooth version of the original discontinous bifurcation diagram of the simple convective heat-salt oscillator studied here.

\section{Conclusions}

Numerical continuation methods were applied to investigate the bifurcation behavior of a simple convective heat-salt oscillator. We found a homoclinic bifurcation and hysteresis behavior that both can be traced back to a multiple bifurcation point in an extended parameter space. We have shown that this point acts as an organizing center for the bifurcation structure and the resulting dynamics. It gives birth to a Hopf bifurcation and a cusp bifurcation. A comparison between the bifurcation behavior described here and the results discussed by Cessi (1996) yields a natural explanation of points 1-3 and a possible explanation of point 4 discussed in the introduction.

The homoclinic bifurcation we identified here is associated with

- arbitrarily long periods close to the breakup of the oscillating regime,

- an abrupt change from a stationary state to a finiteamplitude oscillation that emerges when the freshwater forcing is reduced from high to low values, and

- one extreme phase of the oscillation that resembles the stationary point.

The behavior of the stochastically excited Welander oscillator, as described in Cessi (1996), can be closely related to the bifurcation structure found in this work. The coexistence of a stable fixed point and a stable limits cycle for certain freshwater forcings, which are destroyed by a fold and a homoclinic bifurcation, respectively, result in a stochastically excitable dynamics due to a nonzero jump probability between the attractors in case of parameter noise. Such "excitable systems" have been studied extensively by Stone and Armbruster (1999) and Stone and Holmes (1989) and share typical features of "thermohaline excitability" discussed by Cessi (1996). However, details of the stochastic dynamics are beyond the scope of this bifurcation analysis and will be subject of further work.

Here we have discussed the possibility that, while reducing freshwater forcing, abrupt changes from a stationary nonconvecting state to an oscillating state are to be expected in the vicinity of a homoclinic bifurcation.

While passing the homoclinic threshold (see Fig. 1) the periodicity of the oscillation depends strongly on the freshwater flux (Fig. 2). This specific behavior agrees with the results from a recent study of Timmermann et al. (2003) using a more sophisticated coupled climate model. In that study it is argued that the observed Dansgaard-Oeschger cycles during the last glacial period can be interpreted on the basis of a bifurcation diagram that closely resembles the bifurcation structure presented here.

Hence, we conjecture that observed abrupt changes of the thermohaline circulation may originate from a Hopf bifurcation due to convection. The possibility that a long-term limit cycle can approach a saddle point might also have climatic implications. For the detailed bifurcation structure of the THC advective nonlinearities play a crucial role in order to generate a saddlenode bifurcation. Whether these nonlinarities will 
strongly modify the limit cycle period from its linearly determined value has to be determined.

Furthermore, our work stresses the importance of multiparameter continuation studies of both stable and unstable solutions and of bifurcation points in order to explore the origin of abrupt climate changes.

Acknowledgments. This work is supported by the Deutsche Forschungsgemeinschaft through the Collaborative Research Project SFB460.

\section{REFERENCES}

Bond, G. C., and R. Lotti, 1995: Iceberg discharges into the North Atlantic on millennial timescales during the last glaciation. Science, 267, 1005-1010.

— W. S. Broecker, S. Johnsen, J. McManus, L. Labeyrie, J. Jouzel, and G. Bonani, 1993: Correlations between climate records from North Atlantic sediments and Greenland ice. Nature, 365, 143147.

Cessi, P., 1994: A simple model of stochastically forced thermohaline flow. J. Phys. Oceanogr., 24, 1911-1920.

- 1996: Convective adjustment and thermohaline excitability. $J$. Phys. Oceanogr., 26, 481-491.

Dansgaard, W., and Coauthors, 1984: North Atlantic climatic oscillations revealed by deep Greenland ice cores. Climate Processes and Climate Sensitivity, Geophys. Monogr., Vol. 29, Amer. Geophys. Union, 288-298.

Dijkstra, H., 2000: Nonlinear Physical Oceanography: A Dynamical Systems Approach to the Large Scale Ocean Circulation and El Niño. Kluwer Academic, 480 pp.

Doedel, E. J., and J. P. Kernévez, 1986: AUTO: Software for continuation problems in ode with application. Applied Math Tech. Rep., California Institute of Technology.

Ganopolski, A., and S. Rahmstorf, 2001: Rapid changes of glacial climate simulated in a coupled climate model. Nature, 409, 153158 .

, and - 2002: Abrupt glacial climate changes due to stochastic resonance. Phys. Rev. Lett., 88, doi:10.1103/PhysRev Lett.88.038501.

Gildor, H., and E. Tzipermann, 2001: A sea ice climate switch mechanism for the 100-ky glacial cycles. J. Geophys. Res., 106 (C5), 9117-9133.

Guckenheimer, J., and P. Holmes, 1983: Nonlinear Oscillators, Dynamical Systems, and Bifurcation of Vector Fields. Springer, 459 pp.

Heinrich, H., 1988: Origin and consequences of cyclic ice rafting in the northwest Atlantic Ocean during the past 130000 years. Quat. Res., 29, 143-152.
Johnsen, S. J., and Coauthors, 1992: Irregular glacial interstadials recorded in a new Greenland ice core. Nature, 359, 311-313.

Kuhlbrodt, T., S. Titz, U. Feudel, and S. Rahmstorf, 2001: A simple model for seasonal open ocean convection. Part II: Labrador Sea stability and stochastic forcing. Ocean Dyn., 52, 36-49.

Lindner, B., and L. Schimansky-Geier, 1999: Analytical approach to the stochastic FitzHugh-Nagumo system and coherence resonance. Phys. Rev., 60, 1063-1065.

Lohmann, G., R. Gerdes, and D. Chen, 1996: Stability of the thermohaline circulation in a simple coupled model. Tellus, 48A, $465-476$.

Marotzke, T., and P. H. Stone, 1995: Atmospheric transports, the thermohaline circulation, and flux adjustments in a simple coupled model. J. Phys. Oceanogr., 25, 1350-1364.

Paillard, D., 1995: The hierarchical structure of glacial climatic oscillations: Interactions between ice-sheet dynamics and climate. Climate Dyn., 11, 162-177.

Pikovsky, A., and J. Kurths, 1997: Coherence resonance in a noisedriven excitable system. Phys. Rev. Lett., 78, 775-778.

Rahmstorf, S., 2002: A simple model for seasonal open ocean convection. Part I: Theory. Ocean Dyn., 52, 26-35

Sakai, K., and W. R. Peltier, 1997: A dynamical systems model of the Dansgaard-Oeschger oscillation and the origin of the bond cycle. J. Climate, 12, 2238-2255

Schulz, M., A. Timmermann, and A. Paul, 2002: Relaxation oscillators in concert: A conceptual framework for late Pleistocene millennial-scale climate variability. Geophys. Res. Lett., 29, 2193, doi:10.1029/2002GL016144.

Severinghaus, J. P., and E. J. Brook, 1999: Abrupt climate change at the end of the last glacial period inferred from trapped air in polar ice. Science, 286, 930-934.

Stommel, H. M., 1961: Thermohaline convection with two stable regimes of flow. Tellus, 13, 224-230

Stone, E., and P. Holmes, 1989: Random perturbations of heteroclinic attractors. S.I.A.M. J. Appl. Math., 50, 726-743.

— heteroclinic cycles. Chaos, 9, 499-506.

Timmermann, A., and G. Lohmann, 2000: Noise-induced transitions in a simplified model of the thermohaline circulation. J. Phys. Oceanogr., 30, 1891-1900

— - M. Schulz, H. Gildor, and E. Tziperman, 2003: Coherent resonant millennial-scale climate transitions triggered by massive meltwater pulses. J. Climate, 16, 2569-2585.

Titz, S., T. Kuhlbrodt, and U. Feudel, 2001: Homoclinic bifurcation in an ocean circulation box model. Int. J. Bif. Chaos, 12, 869875 .

- _ - S. Rahmstorf, and U. Feudel, 2002: On freshwater dependent bifurcations in box models of the interhermispheric thermohaline circulation. Tellus, 54A, 89-98.

Welander, P., 1982: A simple heat-salt oscillator. Dyn. Atmos. Oceans, 6, 233-242.

Winton, M., 1993: Deep decoupling oscillations of the oceanic thermohaline circulation. Ice in the Climate System, W. R. Peltier, Ed., NATO ASI Series, Vol. 12, Springer-Verlag, 417-432. 